\title{
New two-colour light curves of Q0957+561: time delays and the origin of intrinsic variations
}

\author{
V. N. Shalyapin ${ }^{1}$, L. J. Goicoechea ${ }^{2}$, E. Koptelova ${ }^{3,4}$, A. Ullán ${ }^{5}$, and R. Gil-Merino ${ }^{6}$
}

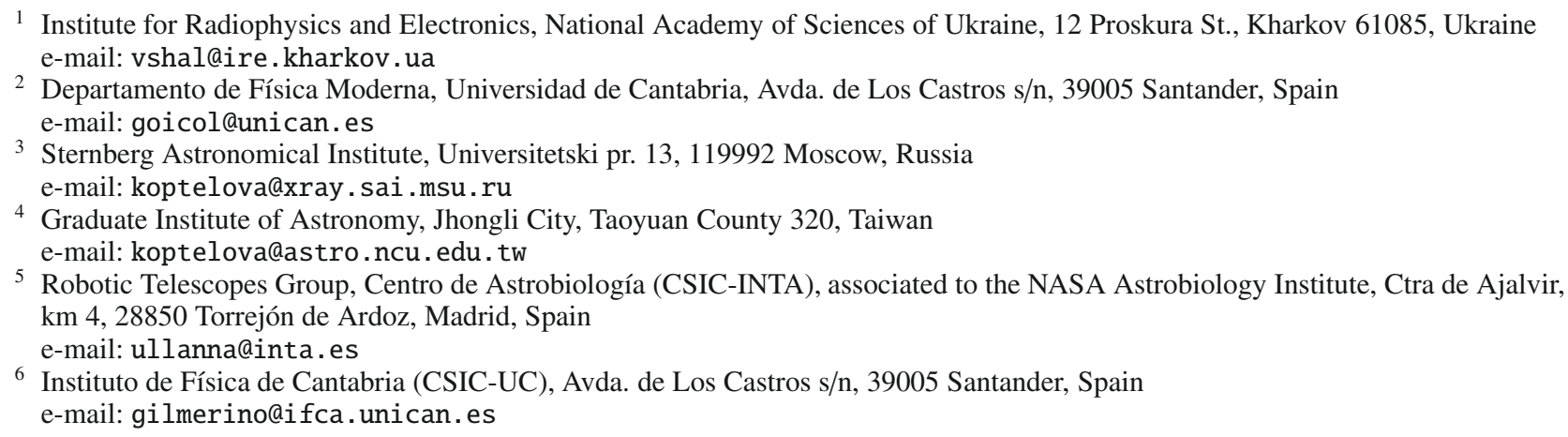

Received 23 June 2008 / Accepted 24 September 2008

ABSTRACT

\begin{abstract}
Aims. We extend the $g r$-band time coverage of the gravitationally lensed double quasar Q0957+561. New $g r$ light curves permit us to detect significant intrinsic fluctuations, to determine new time delays, and thus to gain perspective on the mechanism of intrinsic variability in Q0957+561.

Methods. We use new optical frames of Q0957+561 in the $g$ and $r$ passbands from January 2005 to July 2007. These frames are part of an ongoing long-term monitoring with the Liverpool robotic telescope. We also introduce two photometric pipelines that are applied to the new $g r$ frames of $\mathrm{Q} 0957+561$. The transformation pipeline incorporates zero-point, colour, and inhomogeneity corrections to the instrumental magnitudes, so final photometry to the $1-2 \%$ level is achieved for both quasar components. The two-colour final records are then used to measure time delays.

Results. The $g r$ light curves of Q0957+561 show several prominent events and gradients, and some of them (in the $g$ band) lead to a time delay between components $\Delta t_{B A}=417 \pm 2 \mathrm{~d}(1 \sigma)$. We do not find evidence of extrinsic variability in the light curves of Q0957+561. We also explore the possibility of a delay between a large event in the $g$ band and the corresponding event in the $r$ band. The $g r$ cross-correlation reveals a time lag $\Delta t_{r g}=4.0 \pm 2.0 \mathrm{~d}(1 \sigma$; the $g$-band event is leading) that confirms a previous claim of the existence of a delay between the $g$ and $r$ band in this lensed quasar.

Conclusions. The time delays (between quasar components and between optical bands) from the new records and previous ones in similar bands indicate that most observed variations in Q0957+561 (amplitudes of $\sim 100 \mathrm{mmag}$ and timescales of $\sim 100 \mathrm{~d}$ ) are very probably due to reverberation within the gas disc around the supermassive black hole.
\end{abstract}

Key words. techniques: photometric - gravitational lensing - black hole physics - quasars: individual: Q0957+561

\section{Introduction}

Studies of optical continuum variability in gravitationally lensed quasars (GLQs) have a main advantage: one is usually able to disentangle intrinsic from extrinsic signal in GLQs (e.g., Kundić et al. 1997; Paraficz et al. 2006; Goicoechea et al. 2008, Paper I). Following the original idea by Refsdal (1964), intrinsic variations in brightness records of GLQs have mainly been used to estimate global time delays between components, and to discuss the structure of galaxy mass halos and the expansion rate of the Universe (e.g., Kochanek et al. 2004, and references therein). Less effort has been devoted to investigating the nature of intrinsic fluctuations, which are generated by mechanisms of variability in lensed quasars. This can be done by measuring time delays between components and between optical bands, using prominent events in segments of long-term light curves. Time delays between two given components of a GLQ (determined from different pairs of twin features) arise from gravitational lensing of flares in the variable source. While the flaring of a well-defined emission region (e.g., a ring of the accretion disc) produces a set of similar delays, the existence of flares in some widely separated zones can lead to important time delay differences (Yonehara 1999). For either of the two components, time delays between optical bands (or interband time delays) refer to time lags arising from physical phenomena within the quasar.

The gravitationally lensed double quasar Q0957+561 at $z=$ 1.41 (Walsh et al. 1979) has been monitored photometrically in different optical bands with different telescopes. To derive a global time delay between quasar components, some previous studies used large data sets incorporating all kinds of fluctuations, i.e., noisy or poorly sampled features as well as noticeable gradients and events on several timescales. These large data sets are based on frames that were taken in the 1980s and 90s, and they lead to a global delay of about 423-425 d (Oscoz et al. 2001; Ovaldsen et al. 2003a). The Apache Point Observatory 
(APO) experiment permitted investigators to follow-up the variability in the $g$ and $r$ bands during the 1995 and 1996 seasons, i.e., covering 1.5 years (Kundić et al. 1997). This monitoring programme with the APO $3.5 \mathrm{~m}$ telescope produced accurate light curves of both components Q0957+561A and Q0957+561B, which show sharp intrinsic features with high signal-to-noise ratio $(S / N \geq 3)$. From the APO main twin events in the $g$ band (a prominent event in A and the replica event in B; $S / N \sim 6.5$ ), Kundić et al. (1997) also reported a gravitational lens time delay of $417_{-4}^{+3}$ d $(95 \%$ confidence interval). Complementary to this result, Collier (2001) found that the $r$-band main twin events lag with respect to the ones in the $g$ band by $3.4_{-1.4}^{+1.5} \mathrm{~d}$ (68\% confidence interval), and this interband delay was interpreted as clear evidence for reprocessing in the accretion disc of the quasar.

Goicoechea (2002) reanalysed the APO $g$-band data set to obtain two different gravitational lens time delays of $417.0 \pm 0.6 \mathrm{~d}$ (68\% confidence interval) and $432.0 \pm 1.9 \mathrm{~d}$ (68\% confidence interval) depending on the features taken as reference. The longest delay corresponds to the APO secondary twin events $(S / N \sim 3)$ and it clearly disagrees with the 417-day value. The APO main and secondary twin events in the $g$ band are associated with a main flare and a secondary flare in the variable source, respectively. From the time delay difference of $15 \pm 2$ days ( $68 \%$ confidence interval) and using standard cosmological parameters $(\Omega=0.3, \Lambda=0.7)$, one can also determine a minimum size for the variable source (minimum distance between both flares) of 300 pc (Yonehara 1999; Goicoechea 2002; Yonehara et al. 2003). Several physical sources are consistent with this spatial constraint (from multiple gravitational lens delays) and the interband delay for the main twin events, but either a nuclear accretion disc plus a circumnuclear stellar region or a nuclear accretion disc plus an optical jet are the most probable ones (e.g., Hutchings 2003). We note that the 424-d global time delay between components (Oscoz et al. 2001; Ovaldsen et al. 2003a) coincides with the average of both APO gravitational lens time delays. This suggests the presence of two or more gravitational lens delays in the current large data sets (for alternative explanations, see Schild 2005; Hirv et al. 2007).

In the present study, we substantially extend the $g r$-band time coverage of Q0957+561. The key idea is to use new $g r$ light curves to detect prominent intrinsic events similar to the APO ones. These new features should allow us to determine new time delays and to improve our understanding of the mechanism causing the intrinsic variability. The paper is organised as follows: in Sect. 2, we present new data of Q0957+561 based on recent observations with the Liverpool $2 \mathrm{~m}$ telescope (LT) in the $g$ and $r$ bands, spanning 2.5 years. We describe the observations, the pre-processing, and the photometric procedure for determining calibrated and corrected magnitudes of field stars and quasar components. This last reduction procedure consists of two new pipelines specially designed for the LT. In Sect. 3 we study the time delays between the two components of Q0957+561 as well as the possible delays between the $g$ and $r$ band in the new data set. In Sect. 4 we summarize our results. From the APO and LT delays of Q0957+561, we also discuss the origin of the observed variations with an amplitude of $\sim 100$ mmag and lasting $\sim 100 \mathrm{~d}$.

\section{Data acquisition and reduction}

\subsection{Observations and pre-processing}

Liverpool Quasar Lens Monitoring (LQLM) I is the first phase of an optical follow-up of lensed quasars, undertaken using the
RATCam optical CCD camera on the Liverpool robotic telescope (Steele et al. 2004) between January 2005 and July 2007. The first scientific output of LQLM I was reported in Paper I, and we concentrate here on the observations of Q0957+561 in the $g$ and $r$ filters. The field of view and the pixel scale (binning $2 \times 2$ ) were $\sim 4.6 \times 4$. $^{\prime} 6$ and 0.278 , respectively. The exposure times were 100-200 s ( $g$ band) and $120 \mathrm{~s}$ ( $r$ band). We obtained 286 frames in the $g$ band and 264 frames in the $r$ band. The LT observed for a total science time of $\sim 22.6 \mathrm{~h}$ during the 2.5 -year programme of Q0957+561.

A pre-processing pipeline is applied to all RATCam frames ${ }^{1}$. This performs three basic instrumental reductions: bias subtraction, trimming of the overscan regions, and flat fielding. We also apply a bad-pixel mask (made available by the Angstrom project; Kerins et al. 2006), and correct bad pixels on the CCD. The next step is the pre-selection of frames, based on individual inspection, to assure that exposures verify some elemental conditions (e.g., that the telescope pointing was accurate enough so that the lens system was included in the field of view, that there is no strongly degraded signal, etc.), and that seeing ( $F W H M)$ and sky level (background) values do not exceed reasonable bounds. We only consider frames with $F W H M<3^{\prime \prime}$ due to the separation between the two quasar components (A and B) of $\sim 6^{\prime \prime}$. The pre-selected database contains 199 frames in the $g$ band and 210 frames in the $r$ band. This means that $\sim 75 \%$ of the original LT frames were initially useful.

\subsection{Instrumental photometry}

In a first step, we take a reference frame, i.e., a high-quality frame with small FWHM and large signal-to-noise ratio (SNR). We then measure the positions (with respect to the left bottom corner of the reference frame) of seven reference stars and both quasar images. We select the 7 brightest stars in the Sloan Digital Sky Survey (SDSS) catalog 2 (e.g., Adelman-McCarthy et al. 2007). These stars, having $g$ (SDSS) and $r$ (SDSS) magnitudes below 18 and 17, respectively, were labeled as X, G, F, H, D, E, and R stars in Fig. 1 and Table 1 of Ovaldsen et al. (2003a). Several Image Reduction and Analysis Facility (IRAF) ${ }^{3}$ tasks are also used to identify the available reference objects (in general, less than 7 stars) and the quasar components in the rest of the frames.

In a second step, our photometric pipeline performs aperture photometry of bright field stars and quasar images. This IRAF procedure is used to estimate initial instrumental fluxes

\footnotetext{
1 See the Web site http://telescope.livjm.ac.uk/Info/ TelInst/Inst/RATCam/index . php

2 See the DR6 Catalogue Archive Server site http://cas.sdss. org/astrodr6/en/. Funding for the SDSS has been provided by the Alfred P. Sloan Foundation, the Participating Institutions, the NASA, the NSF, the US Department of Energy, the Japanese Monbukagakusho, and the Max Planck Society. The SDSS is managed by the Astrophysical Research Consortium (ARC) for the Participating Institutions. The Participating Institutions are The University of Chicago, Fermilab, the Institute for Advanced Study, the Japan Participation Group, The Johns Hopkins University, Los Alamos National Laboratory, the Max-Planck-Institute for Astronomy (MPIA), the Max-Planck-Institute for Astrophysics (MPA), New Mexico State University, University of Pittsburgh, Princeton University, the United States Naval Observatory, and the University of Washington.

3 IRAF is distributed by the National Optical Astronomy Observatory, which is operated by the Association of Universities for Research in Astronomy (AURA) under cooperative agreement with the National Science Foundation.
} 

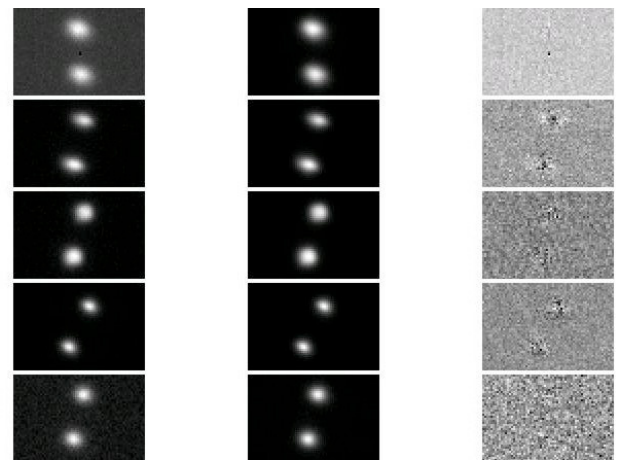

Fig. 1. Observations of Q0957+561 with the Liverpool robotic telescope in the $g$ band. We display system subframes (left panels), model subframes (middle panels), and residual subframes (right panels) for five frames taken during the 2.5-year monitoring period (see main text).

(sources and their associated backgrounds) and to improve the initial source positions on each frame. The pipeline also cuts the original frames in order to produce square subframes with 64 pixels per side: the system subframe (around the centre of the lens system) and subframes of stars (around the bright stars), and makes a PSF subframe containing the clean 2D profile of the $\mathrm{H}$ star (removing the local background). This last empirical PSF is required when performing PSF fitting. The point-like sources (quasar components and stars) are modelled by means of the empirical PSF, whereas the extended source (lensing elliptical galaxy) is modelled by a de Vaucouleurs profile convolved with the empirical PSF. After obtaining all subframes for a given frame, PSF photometry on the stellar and system (crowed field) subframes is performed with IMFITFITS software (McLeod et al. 1998). The pipeline is written in the Python programming language ${ }^{4}$, and incorporates the capabilities of IRAF (through the PyRAF interface) and IMFITFITS, as well as additional numerical and graphical tools.

To determine accurate quasar fluxes, one needs to use a set of constraints. For Q0957+561, the most relevant constraints were obtained from Hubble Space Telescope (HST) frames (Bernstein et al. 1997; Keeton et al. 1998; Kochanek et al. 2008): positions of the B component and the lensing galaxy relative to the A component, and the optical structure of the galaxy, i.e., effective radius, ellipticity, and position angle (a de Vaucouleurs profile was fitted to HST images). Due to the relatively low brightness of the lensing galaxy in the frames and the proximity of the B component to the galaxy, we determine the galaxy-to-H star ratio $(G A L / H)$ in the $g r$ bands from the best LT frames, in terms of $F W H M$ and $S N R$ values. The $\mathrm{H}$ star is relatively bright and it is present in all frames. We then apply the pipeline to all frames (whatever their qualities) in each optical filter, by setting the galaxy fluxes to those derived from the $G A L / H$ ratio and $\mathrm{H}$ star fluxes, and allowing the remaining free parameters to vary.

The photometry pipeline output includes the system subframes, their model subframes (best fits) and the associated residual subframes (system subframes after subtracting model subframes). In Fig. 1 we show system subframes (left panels), model subframes (middle panels), and residual subframes (right panels) corresponding to five frames in the $g$ band. From top to bottom: March 16, $2005\left(F W H M=2{ }^{\prime \prime} 03, S N R=215\right.$, $\left.\chi^{2}=1.44\right)$, November 9, $2005(F W H M=1 \prime \prime 75, S N R=313$, $\left.\chi^{2}=1.32\right)$, April 26, $2006(F W H M=1.76, S N R=371$, $\left.\chi^{2}=2.15\right)$, October 21, $2006(F W H M=1 " .48, S N R=321$,

${ }^{4}$ See the Web site http://www . python.org/

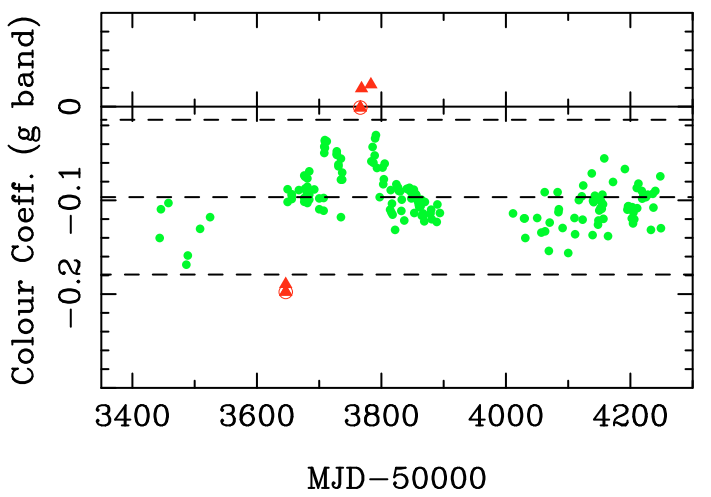

Fig. 2. Colour coefficient in the $g$ band. The values are distributed around the central discontinuous line (average coefficient), and most of them are placed between the top and bottom discontinuous lines (filled circles). Only seven extreme values (triangles and open circles) exceed these limits.

$\left.\chi^{2}=1.30\right)$, and May 28, $2007(F W H M=1 . " 63, S N R=120$, $\left.\chi^{2}=1.43\right)$, where the $S N R$ values are inferred from the A images (having fluxes similar to those of B images) and the $\chi^{2}$ values quantitatively describe the quality of the fits (i.e., these represent the standard reduced $\chi^{2}$ for the best fits). All subframes in Fig. 1 have been expanded by a factor of 2 . The visual comparison between left and middle panels as well as the inspection of patterns of residual brightness (right panels) indicate that the photometric method works well. The pipeline also produces a basic data release file containing values of all relevant instrumental fluxes (stars and quasar images) and relative instrumental magnitudes of both quasar components, e.g., $g_{A}^{*}-g_{E}^{*}$ and $g_{B}^{*}-g_{E}^{*}$ in the $g$ band. To check the reliability of our PSF fitting procedure, we applied a deconvolution technique (Koptelova et al. 2005) to two sets of frames in October-December 2005 ( $g r$ bands). The relative instrumental magnitudes from the deconvolution method agreed with the records from the PSF fitting technique (see Fig. 3 of Goicoechea et al. 2007).

\subsection{Calibrated and corrected magnitudes}

We use a transformation pipeline (in the Python programming language) to obtain SDSS magnitudes from instrumental magnitudes that are corrected for systematic effects. The whole calibration-correction process is outlined in Appendix A. Only frames with $S N R \geq 100$ over Q0957+561A are taken into account (see however Sect. 3). In the $g$ band, this selection leads to 170 frames. In the $r$ band, besides the $S N R$ based selection, the surviving frames from the first season (January-June 2005) are also removed. Several of these $\sim 10 r$-band frames with $S N R$ above 100 (first season) are characterized by an anomalous image formation. Thus, the high-quality data set in the $r$ band includes 167 frames.

The transformation pipeline fits the deviations between instrumental and standard $g$ magnitudes of the 7 reference stars to the transformation model that incorporates a zero-point term $\left(\alpha_{g}\right)$, a colour coefficient $\left(\beta_{g}\right)$, and inhomogeneity coefficients $\left(\gamma_{g, n m}\right)$. Equation (A.11) shows the relationship between the observed magnitude deviation and the model to describe it. The zero-point term and the colour coefficient are allowed to vary over time because the atmospheric and instrumental conditions significantly evolve during the 2.5 years of monitoring. The last ingredient of the model is a linear-quadratic inhomogeneity term, which is related to the $2 \mathrm{D}$ position on the $\mathrm{CCD}$ and tries 


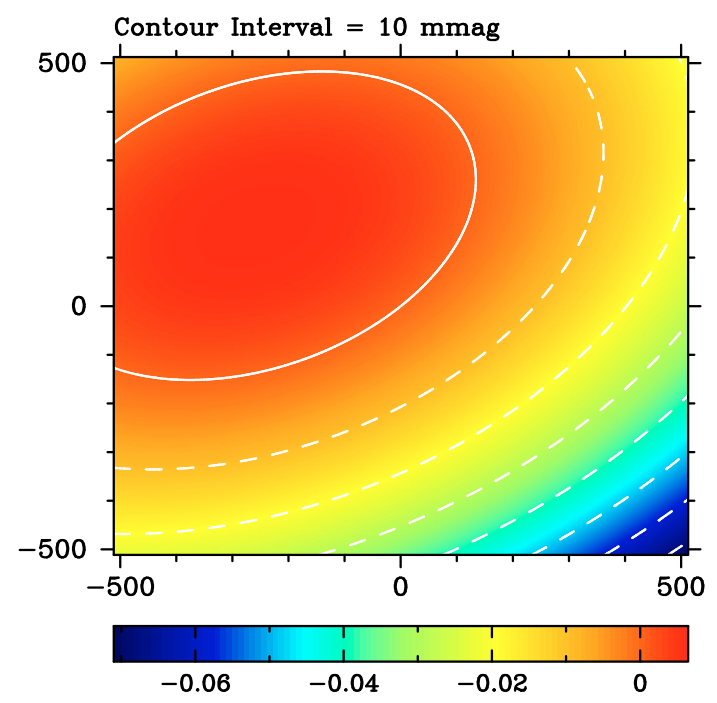

Fig. 3. Inhomogeneity map in the $g$ band. The zero inhomogeneity level is described by means of a continuous line that crosses the centre of the $1024 \times 1024$ camera. Pixels inside this zero level contour have a positive inhomogeneity of a few mmag, whereas the rest of the pixels have a negative inhomogeneity that varies between a few mmag and tens of mmag. We explicitly show contours of $-10,-20,-30,-40,-50,-60$, and -70 mmag.

to correct the possible inhomogeneous response over the camera area (e.g., Manfroid et al. 2001; Magnier \& Cuillandre 2004). Each source occupies different positions on the CCD area during the robotic monitoring period, so this could complicate the collecting of accurate brightness records. With respect to the least squares fit, in Fig. 2 we plot the solution of $\beta_{g}$. The $\beta_{g}$ values are distributed around an average colour coefficient $\left\langle\beta_{g}\right\rangle=-0.097$ (central discontinuous line in Fig. 2), which is close to the typical coefficient (see comments in Appendix A). The scatter is $\sigma\left(\beta_{g}\right)=0.033$, and the $\left\langle\beta_{g}\right\rangle \pm 2.5 \sigma\left(\beta_{g}\right)$ limits also appear in Fig. 2 (top and bottom discontinuous lines). In relation to the average coefficient, there are seven extreme values representing changes from $100 \%$, i.e., values around either -0.2 or 0.0 (triangles and open circles in Fig. 2). The first two triangles and the first open circle correspond to the first night after a realuminisation of the mirror and other maintenance works, and very probably, the telescope was not performing optimally that night. The rest of the extreme values (around day 3770) are associated with dates close to periods of very bad weather. The highest values of $\beta_{q}$ are a consequence of atmospheric-instrumental perturbations during the hard winter in January-February 2006. From the best solutions of $\gamma_{g, n m}$, the pipeline also produces the 2D inhomogeneity pattern, i.e., $\sum_{0<n+m \leq 2} \gamma_{g, n m} x^{n} y^{m}$. This is depicted in Fig. 3. In the transformation procedure, we set the origin of coordinates at the centre of the $1024 \times 1024 \mathrm{CCD}$, so it has an inhomogeneity correction equal to zero. In Fig. 3, we see an inhomogeneity amplitude of $\sim 80 \mathrm{mmag}$, which is consistent with results from other optical telescopes (e.g., Manfroid et al. 2001; Magnier \& Cuillandre 2004). It is evident that the inhomogeneity pattern in Fig. 3 plays a role in achieving 1-2\% photometric accuracy.

After the $g$-band fit, the pipeline computes the calibrated and corrected records of the reference stars and both quasar images from Eq. (A.13). The 14-15th magnitude stars have a typical scatter of 5 mmag, the 15-16th magnitude stars are characterized by a slightly larger scatter of about $7 \mathrm{mmag}$, and the faintest $\sim 18$ th magnitude star ( $\mathrm{R}$ star) has a scatter of about $17 \mathrm{mmag}$.
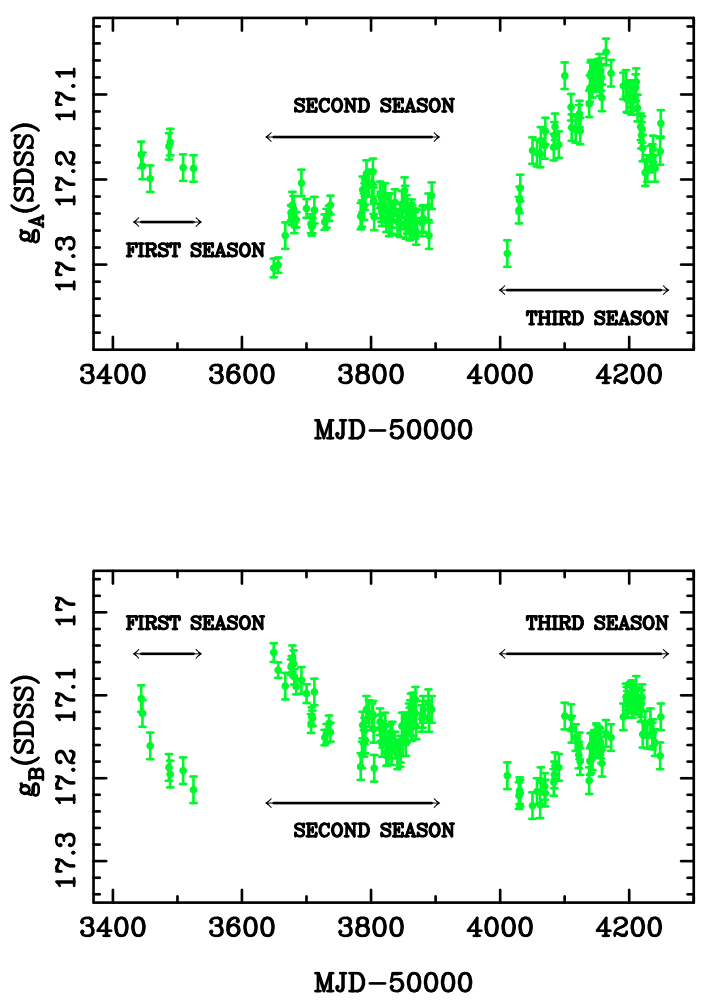

Fig. 4. Final magnitudes of Q0957+561A (top panel) and Q0957+561B (bottom panel) in the $g$ band of the SDSS photometric system. These $g$ SDSS light curves include noticeable fluctuations covering a 2.5-year monitoring period from January 2005 to June 2007.

Although we have several nights with two or three exposures at different times, the standard intranight deviations of the stellar curves do not trace their scatters (see however Paper I). This is not surprising because the intranight variations exclusively correspond to several nights in the second season, which covers a small fraction of the total monitoring period. Thus, after some preliminary test using the stellar records, we find a nonbiased estimator of uncertainties (typical errors): stellar scatters are well traced by the standard deviations between adjacent magnitudes that are separated by $\leq 3 \mathrm{~d}$. As this error estimator gives reasonable results, we apply it to the $g$-SDSS magnitudes of Q0957+561.

A final refinement (selection) is taken into account. Our last selection criterion is colour based: frames with extreme colour coefficients (see the triangles and open circles in Fig. 2) are also removed from the data set. This leads to 163 surviving frames. We obtain uncertainties (see above) of about 16 mmag in both $\sim 17$ th magnitude quasar components, i.e., photometry to the $1-2 \%$ is achieved for the lensed quasar. These typical errors are in complete agreement with the stellar scatters, since they are clearly larger than 5-7 mmag (results for the brightest reference stars) and similar to the result for the faintest reference star R. For each component, we also group pairs or trios of magnitudes measured on the same night. The final light curves of Q0957+561A (top panel) and Q0957+561B (bottom panel) are shown in Fig. 4. These $g$-SDSS light curves include important gradients and prominent events, which resemble those reported by Kundić et al. (1997) using APO observations in the $g$ band. The whole monitoring period consists of three observational seasons: January-June 2005 (first season), October 2005-June 2006 (second season), and October 2006-June 2007 (third season). Besides the three observational seasons, there are two important 

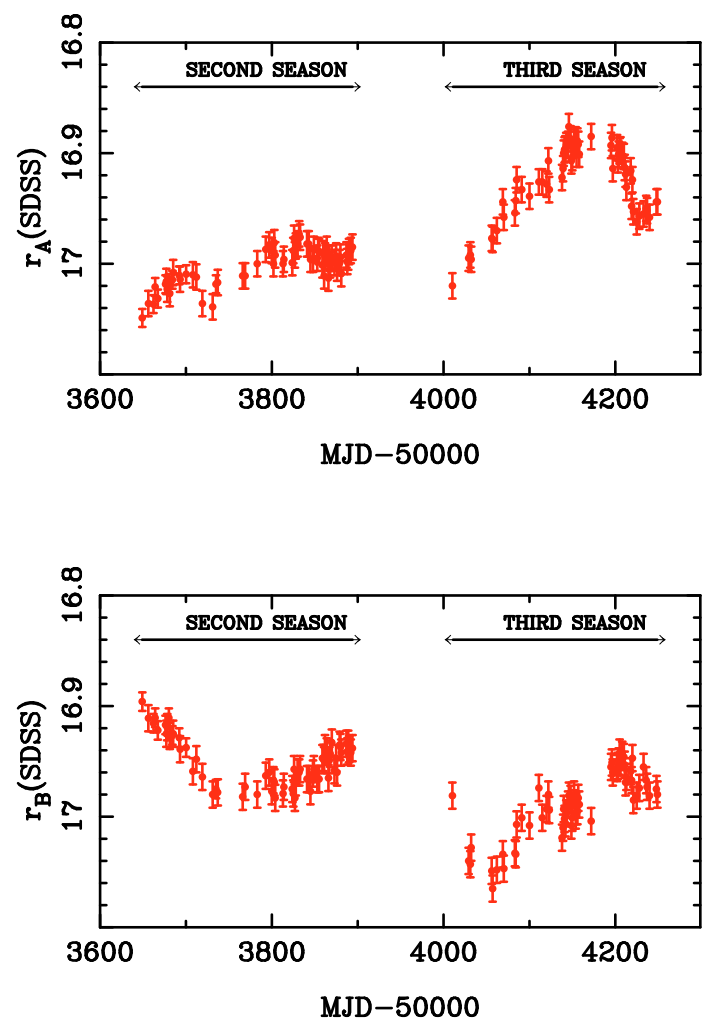

Fig. 5. Final magnitudes of Q0957+561A (top panel) and Q0957+561B (bottom panel) in the $r$ band of the SDSS photometric system. The $r$ SDSS records from October 2005 to June 2007 (two whole seasons) incorporate different prominent features that are also seen in the $g$-SDSS curves (see Fig. 4), with the $g$-SDSS features having a larger amplitude.

gaps in the LT monitoring as a consequence of the annual occultation of the lens system.

The whole procedure in the $g$ band is repeated in the $r$ band. All frames with extreme colour coefficients are not considered in building the final light curves, so we use a data set incorporating 142 frames. With respect to the quasar brightness records, we achieve $\sim 1 \%$ photometry (errors of about $12 \mathrm{mmag}$ ). The final (grouped) magnitudes are presented in Fig. 5, where the top and bottom panels display the records of Q0957+561A and Q0957+561B, respectively. These $r$-SDSS light curves ${ }^{5}$ trace prominent fluctuations that are weaker than the corresponding fluctuations in $g$-SDSS (see Fig. 4 and Sect. 3.2). A similar result was claimed by the APO team (Kundić et al. 1995, 1997), and some evidence for chromatic variability was also suggested by Ullán et al. (2003) (see also the BVRI variations in SerraRicart et al. 1999). The LT records in the red region of the optical spectrum are less noisy than previous curves at red wavelengths (e.g., Kundić et al. 1997; Serra-Ricart et al. 1999), which is due to a combination of an absence of relatively short variations and strict selection procedures.

\section{Time delays of Q0957+561}

\subsection{Delay between quasar components}

The $g$-band light curve of A in the second season (October 2005June 2006) shows significant fluctuations that are repeated in the $g$-band light curve of B during the third season (October

5 The $g r$ records are available at http://grupos.unican.es/ glendama/



Fig. 6. Comparison between the $g$-band light curve of A in the second season (shifted by $420 \mathrm{~d}$; see main text) and the $g$-band light curve of B in the third season. The A record (filled circles) shows two different features separated by a gap of about $50 \mathrm{~d}$ : while the first feature contains an event AE $1 g$ and the beginning of another consecutive event AE2 $g$, the second feature describes the (noisy) decline in flux of AE2 $g$. A vertical line is drawn to distinguish between the two events AE1 $g$ and AE2 $g$. Replica events BE1 $g$ and BE $2 g$ are clearly seen in the B record (open circles).

2006-June 2007). Taking into account the expected delay range of 415-435 d (Kundić et al. 1997; Oscoz et al. 2001; Goicoechea 2002; Ovaldsen et al. 2003a), this result is fully consistent with the presence of intrinsic fluctuations in those records. We use the $g$-SDSS magnitudes of A and B in the second and third seasons, respectively, to accurately measure the time delay(s) between both components of Q0957+561.

About one half of the frames with $80<S N R<100$ correspond to the second season, and thus, they could help to trace the variability of A and to minimize uncertainties in time delay estimates. Their photometric outputs (magnitudes of A) are consistent with results from $S N R \geq 100$ frames at adjacent epochs, so we recover them and expand the $g$-band record of $\mathrm{A}$ in the second season. In Fig. 6, the A light curve, shifted by $420 \mathrm{~d}$ (filled circles), and the unchanged B light curve (open circles) are plotted. A reference value of $420 \mathrm{~d}$ is used to shift in time one component and to compare it with the other (see above and Introduction). The A record shows two different features separated by a gap of about $50 \mathrm{~d}$ (caused by atmospheric-instrumental problems in January-February 2006; see Sect. 2.3). The first feature in the A curve consists of an event AE1 $g$ and the beginning of another consecutive event AE2 $g$, whereas the second feature is a noisy trend associated with the decline in flux of AE $2 g$. These two consecutive events have an amplitude of about 100 mmag and a duration of $50-150 \mathrm{~d}$, and similar fluctuations BE $1 g$ and BE $2 g$ are evident in the $\mathrm{B}$ record.

Firstly, we analyse the twin events AE $1 g$-BE1 $g$ and AE $2 g$ BE2 $g$. The $S / N$ values for them (the ratios between their semiamplitudes and their mean photometric errors) are $(S / N)_{A E 1 g} \sim$ 4 and $(S / N)_{B E 1 g} \sim(S / N)_{B E 2 g} \sim 3.4$. In spite of the fact that AE2 $g$ is a prominent event, it is poorly traced as a consequence of the 50-day gap and the noisy right wing. Thus, we are not able to determine a reliable value of $(S / N)_{A E 2 g}$, and the effective signal-to-noise ratio for AE2 $g$ could be significantly less than $3-4$. The difficulties in inferring a time delay from the AE $2 g$ BE2 $g$ twin events confirm our suspicions. Unfortunately, it is not possible to measure two independent delays, one from AE1g$\mathrm{BE} 1 g$ and the other from AE $2 g$-BE $2 g$. The only options are the estimation of a delay related to the two flares in the source of 
Table 1. Magnitude offset and time delay measurements in the $g$ band.

\begin{tabular}{lccc}
\hline \hline Brightness records & Method & Offset $^{a}$ (mag) & Delay $^{b}(\mathrm{~d})$ \\
\hline A(season 2)-B(season 3) & $\chi^{2}$ & $-0.090 \pm 0.004$ & $417 \pm 2$ \\
& $D^{2}$ & $-0.092 \pm 0.004$ & $416 \pm 5$ \\
AE1-BE1 & $\chi^{2}$ & $-0.083 \pm 0.006$ & $417 \pm 2$ \\
& $\delta^{2}$ & - & $417 \pm 2$ \\
\hline
\end{tabular}

${ }^{a}$ Magnitude offset between the A and B components, where the sign "_" means that A is fainter (see Fig. 6). From $\delta^{2}$ we do not measure the shift in magnitude, since $\delta^{2}$ is a technique based on autocorrelation and cross-correlation functions. All measurements are $1 \sigma$ intervals.

${ }^{b}$ Delay of the replica variation in B with respect to the variation in $\mathrm{A}$ (the A component is leading). All measurements are $1 \sigma$ intervals.

intrinsic variability, i.e., using all events in Fig. 6, or a delay corresponding to the first flare, i.e., from AE1g-BE1 $g$.

Secondly, to calculate the two-flare time delay and magnitude offset (i.e., a constant magnitude shift between the light curves of the two quasar components), we use two techniques: $\chi^{2}$ minimization (e.g., Kundić et al. 1997; Ullán et al. 2006) and the minimum dispersion $\left(D^{2}\right)$ method (Pelt et al. 1994, 1996), characterized by a bin semisize $(\alpha)$ and a decorrelation length $(\delta)$. The choice of $\alpha=\delta=9 \mathrm{~d}$ is a good compromise between the A-B connection and time resolution. Through the $\chi^{2}$ minimization ( $\alpha=9 \mathrm{~d})$, we obtain the best solutions of the delay and magnitudeoffset: $\Delta t_{B A}=417 \mathrm{~d}$ and $\Delta m_{B A}=-0.090 \mathrm{mag}$ $\left(\chi^{2} \sim 1.2\right)$. The sign "-" in the $\Delta m_{B A}$ value means that the A component is fainter. The $D^{2}$ minimization $(\delta=9 \mathrm{~d})$ gives the best solutions of $\Delta t_{B A}=416 \mathrm{~d}$ and $\Delta m_{B A}=-0.092 \mathrm{mag}$. The uncertainties in the magnitude offset and time delay are inferred from 1000 repetitions of the experiment (synthetic light curves based on the observed records). The $1 \sigma$ intervals appear in Table 1. Table 1 indicates that the error in time delay from the $\chi^{2}$ minimization is substantially less than the error from the minimum dispersion method. Both measurements of the twoflare time delay are consistent with the APO main delay in the $g$ band (see Introduction).

Thirdly, we exclusively use the AE $1 g$-BE $1 g$ twin events. The idea is to accurately measure the gravitational lens delay associated with only one flare produced in the source of variability. This time we focus on the $\delta^{2}$ method (see, e.g., Paper I) and the $\chi^{2}$ minimization, which produces a delay error smaller than the delay uncertainty from the minimum dispersion technique (see Table 1). The $\delta^{2}$ technique obtains the optimal match between the time-shifted discrete autocorrelation function $(D A F)$ and the discrete cross-correlation function ( $D C F$; Edelson \& Krolik 1988). From the $\chi^{2}$ minimization $(\alpha=9 \mathrm{~d})$, the best solutions of the time delay and magnitude offset are $417 \mathrm{~d}$ and $-0.083 \mathrm{mag}$, respectively $\left(\chi^{2} \sim 0.9\right)$. From the $\delta^{2}$ method and 1000 synthetic light curves, the delay measurement ( $1 \sigma$ interval) is identical to that derived from the $\chi^{2}$ technique (see Table 1). Therefore, the LT first twin events are useful to determine a robust time delay $\Delta t_{B A}=417 \pm 2 \mathrm{~d}(1 \sigma)$. This is fully consistent with the APO main delay (Kundić et al. 1997; Goicoechea 2002). The $\delta^{2}$ analysis also indicates that $\Delta t_{B A} \leq 424 \mathrm{~d}(99 \%$ confidence interval), so the AE1 $g$-BE1 $g$ delay is inconsistent (at about the $3 \sigma$ level) with the APO secondary delay (Goicoechea 2002). The $r$-band curves of AE1-BE1 are not used to determine a time delay because $S / N<3$ for these twin events (e.g., Pijpers 1997).

We now check for the possible existence of extrinsic variability in our records. We compute the difference light curve between the A and B components, since no extrinsic variability
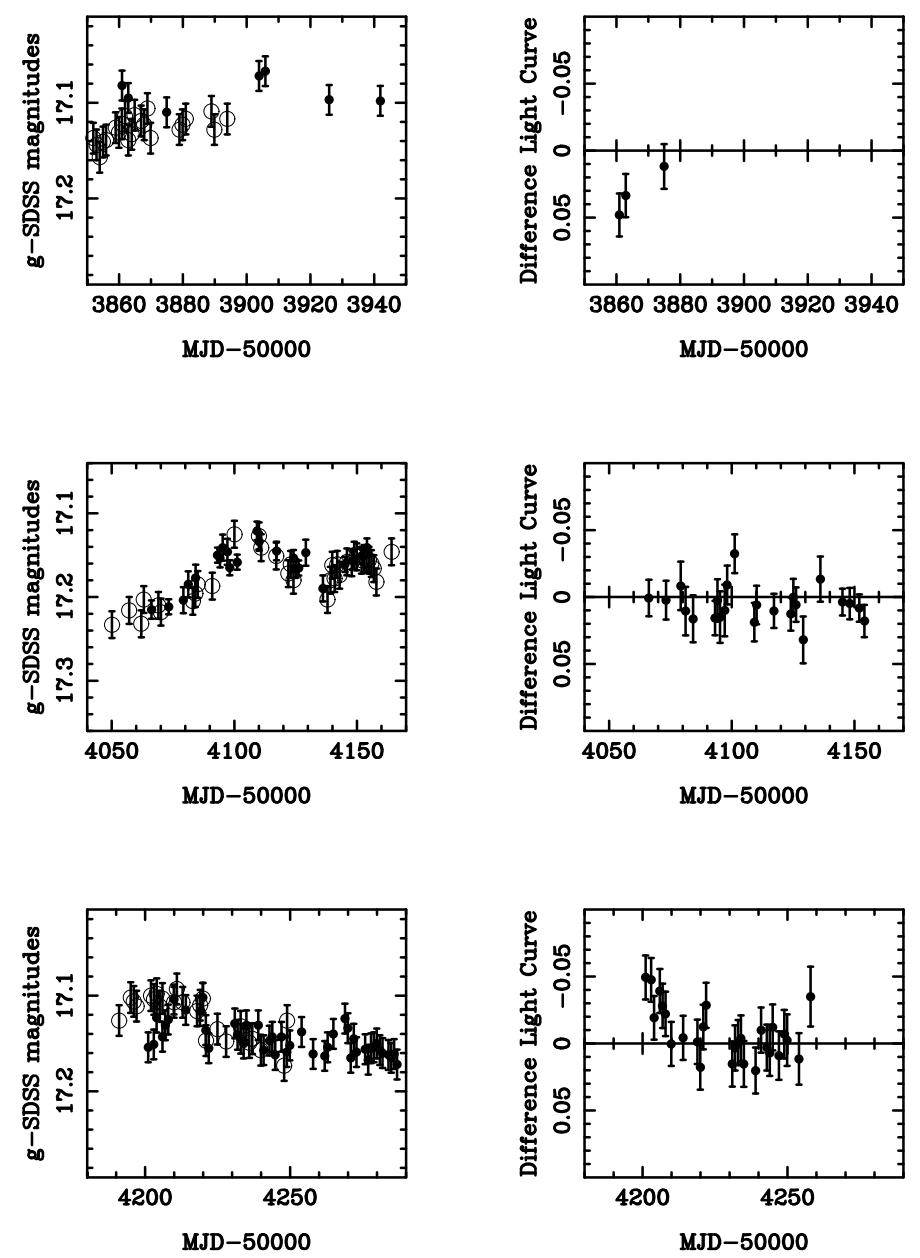

Fig. 7. Overlapping periods and difference light curves in the $g$ band. We show the overlap between the A (filled circles) and B (open circles) whole records, when the A magnitudes are shifted by the best solutions of the time delay and the magnitude offset (left panels). We also draw the difference light curve (right panels). The three overlap periods cover $\sim 20 \mathrm{~d}$ (top panels), $\sim 90 \mathrm{~d}$ (middle panels), and $\sim 60 \mathrm{~d}$ (bottom panels).

should result in a flat difference light curve. To obtain the difference light curve, the magnitude- and time-shifted light curve of image A is subtracted from the light curve of image B (e.g., Schmidt \& Wambsganss 1998; Gil-Merino et al. 2001). In Fig. 7 (left panels), we show the overlap between the A (filled circles) and B (open circles) whole records, when the A magnitudes are shifted by the best solutions of the time delay and the magnitude offset. The difference light curve is also plotted in the right panels of Fig. 7. The overlap between A-first season and B-second season covers a very short period of about $20 \mathrm{~d}$ (see the top panels of Fig. 7). For this overlap period, the difference curve contains two consecutive deviations from the zero level, which are not significative (e.g., Gil-Merino et al. 2001). The overlap between A-second season and B-third season is much more important than the first overlap (in the top panels). In the middle panels of Fig. 7, we display the situation before the 50-day gap (see above and Fig. 6), where the difference curve has a noisy trend that is consistent with zero. In the bottom panels, the behaviour after the 50-day gap is shown. In this last period, the difference curve is also mainly noise. However, a clear event appears at the beginning of the overlapping period, i.e., six consecutive points are placed above the zero level. Although this naively could be interpreted as the wing of a microlensing event (i.e., extrinsic 
variability), the A data were obtained at the end of a hard winter in which the colour coefficient strongly deviated (40-70\%) from its average value. While some frames with extreme colour coefficients are not considered in the analysis (see the triangles and the open circle around day 3770 in Fig. 2), additional adjacent frames are also unsuitable for fine variability studies. Therefore, bad weather and anomalous behaviour of the LT devices are the most reasonable explanations for the anomalous variation in $\mathrm{A}$ that is simultaneously observed in both components. In summary, we do not find evidence of extrinsic variability in the light curves of Q0957+561.

\subsection{Delay between optical bands}

The time delay between optical-UV continuum flux variations at two different wavelengths can be used to test the variability secenario (e.g., Collier et al. 1999). It might be produced by reprocessing of high energy radiation in an accretion disc around a supermassive black hole. The reprocessing hypothesis assumes that the optical-UV variations are the response of the gas in the disc to higher-energy fluctuations in the vecinity of the disc axis. Moreover, the existence of a radiative coupling between the variations is also assumed, i.e., the time delay represents a light-travel time between two disc annuli (see details in Collier et al. 1999). Collier et al. (1999) measured two time lags between fluctuations at two optical wavelengths and the corresponding UV fluctuations (UV variability leading optical variations) in the records of NGC 7469 at $z=0.016$, and they found a good agreement between their delay estimates $(\sim 1-2 \mathrm{~d})$ and reverberation within an accretion disc. Sergeev et al. (2005) and Cackett et al. (2007) also explored the thermal reprocessing hypothesis in local active galactic nuclei. For Q0957+561, Collier (2001) reported a delay of about $3.4 \mathrm{~d}$ between the $r$-band and $g$-band APO main events ( $g$-band events leading those in the $r$ band), which translates into a rest-frame lag of about $1.4 \mathrm{~d}$, in excellent agreement with predictions of the disc reprocessing scenario. This first delay between optical bands for a GLQ requires an independent confirmation as well as new efforts with other GLQs (e.g., Koptelova et al. 2006), and here we try to reach the first goal.

For such a task, we focus on the LT events with highest $S / N$. The AE1-BE1 twin events are ruled out because $(S / N)<3$ in the $r$ band. However, there are two very prominent variations around day 4150 in the top panels of Figs. 4, 5 (A component). These $\mathrm{AE} 3 g$ and $\mathrm{AE} 3 r$ variations last $\sim 250 \mathrm{~d}$ (the whole light curves of $\mathrm{A}$ in the third season are considered as large events) and have signal-to-noise ratios above 6 . We use fluxes in arbitrary units $f=10^{7} \times 10^{-0.4 m}$ to compare AE $3 g$ and AE $3 r$. The use of fluxes (instead of magnitudes $m$ ) permits a fair cross-correlation between two records that, apart from a possible delay, differ in a multiplicative constant and an additive constant. On average, the light curves were sampled two times per week. However, there are 20-day gaps around day 4180. Unfortunately, due to a combination of the kind of variability (time asymmetric events consisting of slow rises and rapid declines) and these short gaps close to the maxima, it is not possible to infer a reliable $D C F$ with good time resolution, i.e., $\alpha \leq 10 \mathrm{~d}$ (see above). For $\alpha<20 \mathrm{~d}$, 20 -day artifacts at lags of $\pm 50 \mathrm{~d}$ appear in the $D C F$. This unphysical signal at $\pm 50 \mathrm{~d}$ is only avoided using longer bins with $\alpha \geq 20 \mathrm{~d}$, so we are forced to take relatively long bins. This is not a problem at all, but the measurement would be more accurate (but not more reliable) with better time resolution. Some $D C F$ (filled circles) and $\langle D A F\rangle$ (open circles) trends are shown in Fig. 8. The top, middle, and bottom panels of Fig. 8 contain
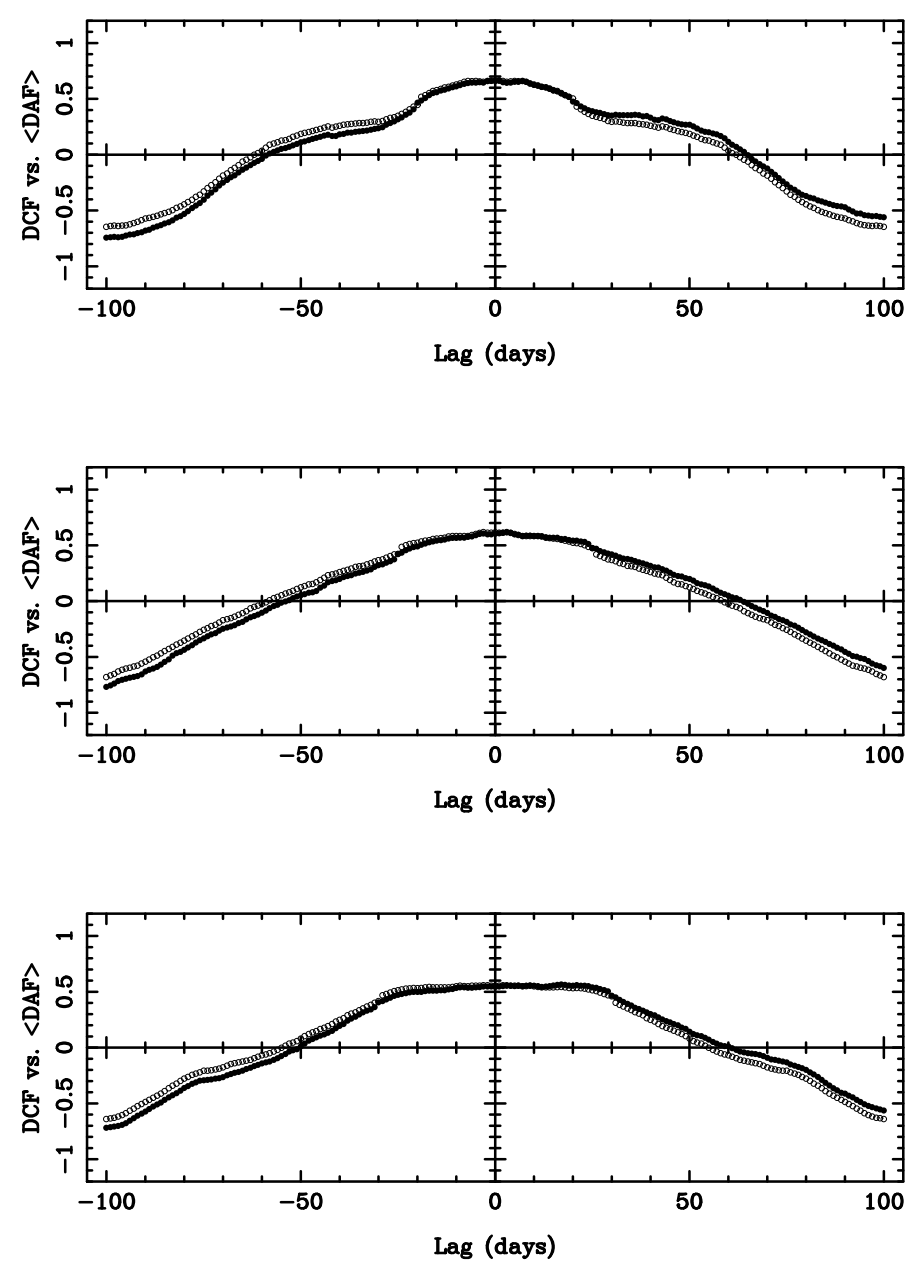

Fig. 8. Comparison between the $D C F$ (filled circles) and the $\langle D A F\rangle$ (open circles). Whilethe $D C F$ is the $g r$ cross-correlation function, the $\langle D A F\rangle$ is the average of the $g g$ and $r r$ autocorrelation functions. We use the AE3 $g$-AE3 $r$ events (see main text) and three bin semisizes: $\alpha=20$ (top panel), 25 (middle panel), and 30 (bottom panel) d.

the results for $\alpha=20,25$, and $30 \mathrm{~d}$, respectively. Here, $\langle D A F\rangle$ is the average of the $g g$ and $r r$ autocorrelation functions, whereas $D C F$ represents the $g r$ cross-correlation function.

In Fig. 8, there are no important distortions in the features of the $D C F$ compared to the features in the $\langle D A F\rangle$, but the existence of a delay of several days is evident. In other words, to get an optimal match, the $\langle D A F\rangle$ should be shifted by several days. Possible values of this time shift $(\theta)$ versus the associated $\delta^{2}(\theta)$ values normalised by its minimum value $\delta^{2}\left(\theta_{0}\right)$ are plotted in Fig. 9. The $\delta^{2}(\theta)$ function was defined in Eq. (7) of Serra-Ricart et al. (1999) (see also above), and we use $\alpha=20$ (dotted line), 25 (dashed line), and 30 (solid line) d in Fig. 9. This figure displays relatively narrow peaks centered on 3-4.5 d (best values of the interband delay; AE3 $g$ leading AE3r). Uncertainties are again computed by applying the $\delta^{2}$ minimization to 1000 synthetic data sets. Through the distributions of delays ( $\alpha=20-40 \mathrm{~d})$, five $1 \sigma$ measurements are presented in Table 2. The $\delta^{2}$ results in Table 2 agree with the previous time lag determination from APO light curves, and we adopt $\Delta t_{r g}=4.0 \pm 2.0 \mathrm{~d}$ (using an intermediate bin semisize $\alpha=30 \mathrm{~d}$; the probability of $\Delta t_{r g} \leq 0$ is only $7.5 \%$ ) as our final $1 \sigma$ measurement. 


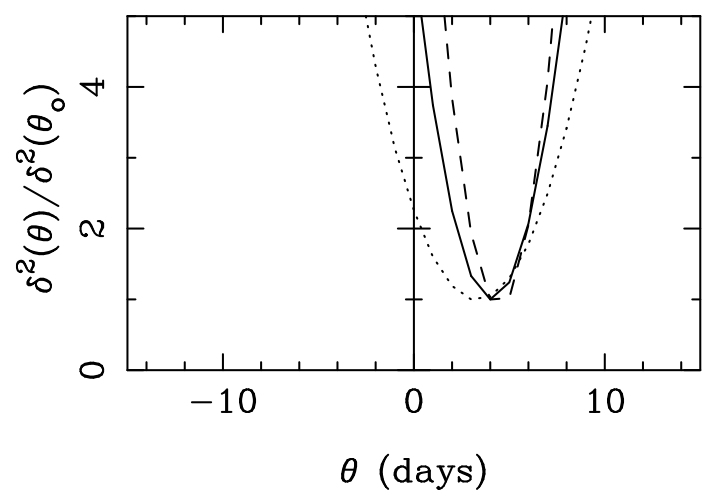

Fig. 9. Normalised $\delta^{2}$ function from the AE $3 g-\mathrm{AE} 3 r$ events. We use bin semisizes $\alpha=20$ (dotted line), 25 (dashed line), and 30 (solid line) d. In Fig. 8, we can observe the presence of time shifts between the $D C F$ and $\langle D A F\rangle$, which translate into interband delay peaks centered on 3-4.5 d (AE3g leading $\mathrm{AE} 3 r$ ).

Table 2. Time lag measurements from the AE $3 g$-AE3 $r$ events.

\begin{tabular}{ccc}
\hline \hline$\alpha^{a}(\mathrm{~d})$ & ${\text { Time } \operatorname{lag}^{b}(\mathrm{~d})}$ & Probability of lags $\leq 0(\%)$ \\
\hline 20 & $3.0 \pm 2.0$ & 11.6 \\
25 & $4.5 \pm 2.5$ & 6.9 \\
30 & $4.0 \pm 2.0$ & 7.5 \\
35 & $3.5 \pm 2.0$ & 8.5 \\
40 & $3.5 \pm 2.0$ & 9.3 \\
\hline
\end{tabular}

${ }^{a}$ We use the $\delta^{2}$ technique (see main text) and five values of the bin semisize $\alpha .{ }^{b}$ All measurements are $1 \sigma$ intervals, and positive lags mean that the $r$-band event is delayed in relation to the arrival of the associated $g$-band event.

\section{Summary and conclusions}

Liverpool Quasar Lens Monitoring is a long-term project to follow the optical (gri bands) variability of 10-20 GLQs with the Liverpool robotic telescope (Steele et al. 2004). The first phase of this project (LQLM I) was conducted between January 2005 and July 2007. While in Paper I we mainly studied the intrinsic variability of Q0909+532 in the $r$ band, in this paper we present the monitoring programme of Q0957+561 in the $g r$ bands. A main goal of our project (LQLM) is to considerably increase the public database of GLQs. Thus, all LQLM I pre-processed frames of Q0909+532 and Q0957+561 are publicly available on the Lens Image Archive of the German Astrophysical Virtual Observatory ${ }^{6}$.

We have fully developed two photometric pipelines through the 3 years of observations and analyses. The transformation pipeline incorporates zero-point, colour, and inhomogeneity corrections in the instrumental fluxes, so photometry to the $1-2 \%$ is achieved for Q0957+561A and Q0957+561B. We detect an inhomogeneous response over the CCD area, which has an amplitude of $\sim 80$ mmag (from maximum to minimum) and is consistent with studies in other optical telescopes (e.g., Manfroid et al. 2001; Magnier \& Cuillandre 2004). Moreover, the colour coefficient is allowed to vary through time, because the atmospheric-instrumental conditions signicantly evolve through 2.5 years of monitoring. Due to atmospheric-instrumental problems at some epochs, the colour coefficient reaches anomalous values, i.e., we obtain dramatic deviations with respect to the

\footnotetext{
6 See the Web site http://vo.uni-hd.de/lensdemo/view/q/ form
}

average coefficient. Thus, the frames corresponding to an anomalous coefficient are removed or not considered.

The LT $g r$ light curves of Q0957+561 show several prominent events and gradients, and some of them (in the $g$ band) are used to infer a time delay between components $\Delta t_{B A}=417 \pm 2 \mathrm{~d}$ $(1 \sigma)$. This gravitational lens delay from new $g$-band events is in agreement with the delay from the previous APO $g$-band main events (Kundić et al. 1997), so the associated UV flares in the variable source (APO and LT events) probably originate in the same emission region (Yonehara 1999). Taking into account that the previous APO $g r$-band main events are plausibly due to reverberation within an irradiated accretion disc (Collier 2001), the new $g r$-band events are likely related to flares in the central accretion disc. In addition, the delay between the two new LT large events in the $g$ and $r$ bands: $\Delta t_{r g}=4.0 \pm 2.0 \mathrm{~d}(1 \sigma$; the $g$-band event is leading), coincides with the estimation by Collier (2001) and agrees with flares generated during reprocessing in the accretion disc. Therefore, most APO-LT variations in the $g$ and $r$ bands are very probably associated with the gas disc around the supermassive black hole (only the APO secondary events have been associated with flares that were produced far away from the accretion disc; see Introduction). The detection of the same interband delay (between the $g$ and $r$ band) in the two monitoring campaigns (APO and LT) also suggests that the accretion disc reprocessing in Q0957+561 is a usual occurance at different times for different prominent flares. Hence, very likely, most observed variations in the $g$ and $r$ bands (APO and LT fluctuations with an amplitude of $\sim 100$ mmag and lasting 100 d) are associated with reverberation within the gas disc around the supermassive black hole.

We add 2.5 years of time coverage to the previous 1.5 -year gr-band records of Q0957+561, and remark that our difference light curves are consistent with zero. Thus, there is no evidence of extrinsic variations in both APO and LT independent experiments separated by $\sim 10$ years. These results disagree with the claim by Schild (2005) that microlenses in the lensing galaxy affect the observed variability. Therefore, the complex quasar structure suggested by this author is not supported by the $\mathrm{gr}$ band light curves of Q0957+561. We also remark that double replicas in the records of the B component are not detected in the APO and LT experiments. This clearly contradicts previous conclusions by Hirv et al. (2007), which indicated that the full B light curve of the lensed quasar can be decomposed into a sum of two similar and time shifted curves. Finally, the APO-LT combined database of Q0957+561 (together with other monitorings done between both experiments) is a promising tool for studying the quasar structure and the composition of the lensing halo (e.g., Schmidt \& Wambsganss 1998; Kochanek 2004).

Acknowledgements. We thank an anonymous referee for several comments that improved the presentation of our results. We also thank C. Moss for guidance in the preparation of the robotic monitoring project with the Liverpool telescope. The Liverpool Telescope is operated on the island of La Palma by Liverpool John Moores University in the Spanish Observatorio del Roque de los Muchachos of the Instituto de Astrofisica de Canarias with financial support from the UK Science and Technology Facilities Council. We thank B. McLeod for providing the IMFITFITS software to us. We use information taken from the Sloan Digital Sky Survey (SDSS) Web site, and we are grateful to the SDSS team for doing that public database. This research has been supported by the Spanish Department of Education and Science grants AYA2004-08243-C03-02 and AYA2007-67342-C03-02, University of Cantabria funds, grant for young scientists of the President of the Russian Federation (number MK-2637.2006.2), Deutscher Akademischer Austausch Dienst (DAAD) grant number A/05/56557 and grant of Russian Foundation for Basic Research (RFBR) 06-02-16857. EK holds the Taiwan National Research Councils grant No. 96-2811-M-008-058. RGM holds a grant of the ESP2006-13608-C02-01 project financed by the Spanish Department of Science and Innovation. 


\section{Appendix A: Transformation equations}

The initial transformation equations for a given reference star are

$g^{*}\left(t_{j}\right)=g+A_{g}\left(t_{j}\right)+C_{g}\left(t_{j}\right)(g-r)$,

$r^{*}\left(t_{k}\right)=r+A_{r}\left(t_{k}\right)+C_{r}\left(t_{k}\right)(r-i)$,

where $g^{*}$ and $r^{*}$ are the instrumental magnitudes of the star, $g, r$, and $i$ are its standard magnitudes, $A_{g}$ and $A_{r}$ are the zero-point terms (including instrumental and atmospheric corrections), and $C_{g}$ and $C_{r}$ are the colour coefficients. The zero-point terms and the colour coefficients are expected to significantly change during the 2.5-year monitoring period, so we explicitly consider their time evolution. Here, $t_{j}$ and $t_{k}$ denote observation times in the $g$ and $r$ bands, respectively. As we are initially interested in the usual systematic corrections, Eqs. (A.1, A.2) do not include other possible terms (see here below). Instead of the LT photometric system (ugriz $\equiv u^{\prime} g^{\prime} r^{\prime} i^{\prime} z^{\prime}$ ), we want to use the SDSS "natural" system, since accurate standard magnitudes are available in this SDSS $2.5 \mathrm{~m}$ system (e.g., Smith et al. 2002; Stoughton et al. 2002). SDSS magnitudes are also suitable for comparing our results with future data of Q0957+561 using different facilities and/or SDSS quasar studies/databases (e.g., Vanden Berk et al. 2004; Schneider et al. 2007). From equations for transforming LT magnitudes to magnitudes in the SDSS system $^{7}$ :

$g=g_{\mathrm{SDSS}}+B_{g}(g-r)+K_{g}$,

$r=r_{\mathrm{SDSS}}+B_{r}(r-i)+K_{r}$

and equations that relate LT and SDSS colours:

$g-r=a_{g r}(g-r)_{\mathrm{SDSS}}+b_{g r}$,

$r-i=a_{r i}(r-i)_{\mathrm{SDSS}}+b_{r i}$,

it is possible to rewrite Eqs. (A.1, A.2) as

$g^{*}\left(t_{j}\right)=g_{\mathrm{SDSS}}+\alpha_{g}\left(t_{j}\right)+\beta_{g}\left(t_{j}\right)(g-r)_{\mathrm{SDSS}}$,

$r^{*}\left(t_{k}\right)=r_{\mathrm{SDSS}}+\alpha_{r}\left(t_{k}\right)+\beta_{r}\left(t_{k}\right)(r-i)_{\mathrm{SDSS}}$.

The $\alpha_{g}$ term and the $\beta_{g}$ coefficient are given by (it is trivial to write expressions for $\alpha_{r}$ and $\beta_{r}$ )

$\alpha_{g}\left(t_{j}\right)=A_{g}\left(t_{j}\right)+K_{g}+b_{g r}\left[B_{g}+C_{g}\left(t_{j}\right)\right]$,

$\beta_{g}\left(t_{j}\right)=a_{g r}\left[B_{g}+C_{g}\left(t_{j}\right)\right]$.

Taking into account typical values of $C_{g}(\sim-0.029)$ and $C_{r}$ $(\sim 0.034)$ reported by the LT team (on the LT Web site; see main text), the SDSS estimates of $B_{g}(\sim-0.060)$ and $B_{r}(\sim-0.035)$ on the SDSS Web site (see here above), and $a_{g r} \sim a_{r i} \sim 1$, we expect typical colour coefficients $\beta_{g} \sim-0.089$ and $\beta_{r} \sim-0.001$. On the other hand, the adopted standard magnitudes of the reference stars (XGFHDER field stars; see main text) appear in Table A.1. These are PSF magnitudes in the SDSS catalogue ${ }^{2}$.

In order to achieve $1-2 \%$ photometric accuracy with the RATCam camera (on the LT), one additional detail must be taken into account in the transformation Eqs. (A.7, A.8). We introduce an inhomogeneity term that corrects the flat-field systematic error over the camera area, which might have a total amplitude of 50 mmag (e.g., Manfroid et al. 2001; Magnier \& Cuillandre 2004). For example, this kind of error could be related to twilight flats. During twilight exposures, some scattered light (within the dome) would reach the camera, and thus, the illumination would not be homogeneous. This effect invalidates the basic hypothesis

\footnotetext{
${ }^{7}$ See the Web site http://www.sdss.org/dr6/algorithms/
}

Table A.1. Adopted standard magnitudes of the reference stars.

\begin{tabular}{lccc}
\hline \hline Star & $g_{\text {SDSS }}$ & $r_{\text {SDSS }}$ & $i_{\text {SDSS }}$ \\
\hline $\mathrm{X}$ & 14.213 & 13.849 & 13.750 \\
$\mathrm{G}$ & 14.461 & 14.157 & 14.060 \\
$\mathrm{~F}$ & 14.513 & 14.186 & 14.089 \\
$\mathrm{H}$ & 15.116 & 14.422 & 14.174 \\
$\mathrm{D}$ & 15.485 & $14.951^{a}$ & 14.770 \\
$\mathrm{E}$ & 15.816 & 15.217 & 15.018 \\
$\mathrm{R}$ & 17.879 & 16.801 & 16.419 \\
\hline
\end{tabular}

a The SDSS catalogue seems to contain a wrong value of the $r$-SDSS magnitude of the D star $\left(r_{\mathrm{SDSS}}=15.674\right)$, so the $\mathrm{D}$ star would be fainter than the E star in this band. This disagrees with our current LT observations and several previous observations in the red region of the optical spectrum. Thus, the $r$-SDSS magnitude is inferred through the $r_{\text {SDSS }}$ vs. $V R$ relationship: $r_{\mathrm{SDSS}}=V-0.89(V-R)+0.39$. This law is based on the $r$-SDSS magnitudes of the rest of stars and the corresponding $V R$ magnitudes in Tables 1, 2 of Ovaldsen et al. (2003b).

of homogeneous illumination. Here, we assume a second order 2D polynomial to account for the inhomogeneity term, so the final transformation equations are

$$
\begin{aligned}
g^{*}\left(t_{j}\right)= & g_{\mathrm{SDSS}}+\alpha_{g}\left(t_{j}\right)+\beta_{g}\left(t_{j}\right)(g-r)_{\mathrm{SDSS}} \\
& +\sum_{0<n+m \leq 2} \gamma_{g, n m} x^{n}\left(t_{j}\right) y^{m}\left(t_{j}\right) \\
r^{*}\left(t_{k}\right)= & r_{\mathrm{SDSS}}+\alpha_{r}\left(t_{k}\right)+\beta_{r}\left(t_{k}\right)(r-i)_{\mathrm{SDSS}} \\
& +\sum_{0<n+m \leq 2} \gamma_{r, n m} x^{n}\left(t_{k}\right) y^{m}\left(t_{k}\right)
\end{aligned}
$$

where $(x, y)$ is the $2 \mathrm{D}$ position of the star on the CCD. To find the relevant parameters in the $g$ band, i.e., $\alpha_{g}\left(t_{j}\right), \beta_{g}\left(t_{j}\right)$, and $\gamma_{g, n m}$, we may fit the observed magnitude deviations (instrumental standard) of the seven reference stars to the model incorporating the three systematic terms: zero-point, colour, and inhomogeneity. Once the fit has been made, the $g$-SDSS magnitude of any point-like source (star or quasar) is derived in a straightforward way:

$$
\begin{aligned}
g(S D S S)= & g_{\mathrm{SDSS}}+\delta=g^{*}\left(t_{j}\right)-\alpha_{g}\left(t_{j}\right)-\beta_{g}\left(t_{j}\right)(g-r)_{\mathrm{SDSS}} \\
& -\sum_{0<n+m \leq 2} \gamma_{g, n m} x^{n}\left(t_{j}\right) y^{m}\left(t_{j}\right)
\end{aligned}
$$

In Eq. (A.13), $\delta$ represents the deviation caused by random noise (e.g., photon noise) and unkown (but presumibly small) systematic corrections. For a non-variable star (e.g., a reference star), variations in $g($ SDSS $)$ are generated by noise $(\delta)$. However, for variable stars or quasars, there are two kinds of variability. While true variability is due to time evolution of $g_{\text {SDSS }}$, noise is an additional source of fluctuations. The $(g-r)_{\text {SDSS }}$ colour of Q0957+561 might also evolve over time. Thus, the use of an average colour $\left\langle(g-r)_{\mathrm{SDSS}}\right\rangle$ in the colour correction introduces a systematic noise $\delta_{\text {col }}=\beta_{g}\left(t_{j}\right) \delta(g-r)_{\text {SDSS }}$ associated with the colour variation. Fortunately, for moderate fluctuations with amplitude of $\sim 25$ mmag (e.g., Kundić et al. 1995), the amplitude of the colour noise is only $\sim 0.2 \%$, well below our accuracy goal. The $r$-SDSS magnitude of a source is given by an expression similar to Eq. (A.13).

\section{References}

Adelman-McCarthy, J. K., Jennifer, K., Agüeros, Marcel A., et al. 2007, ApJS, 172,634

Bernstein, G., Fischer, P., Tyson, J. A., \& Rhee, G. 1997, ApJ, 483, L79 
Cackett, E. M., Horne, K. \& Winkler, H. 2007, MNRAS, 380, 669

Collier, S. 2001, MNRAS, 325, 1527

Collier, S., Horne, K., Wanders, I., \& Peterson, B. M. 1999, MNRAS, 302, L24

Edelson, R. A., \& Krolik, J. H. 1988, ApJ, 333, 646

Gil-Merino, R., Goicoechea, L. J., Serra-Ricart, M., et al. 2001, MNRAS, 322 , 397

Goicoechea, L. J. 2002, MNRAS, 334, 905

Goicoechea, L. J., et al. 2007, in Highlights of Spanish Astrophysics IV, ed. F. Figueras, J. M. Girart, M. Hernanz, \& C. Jordi (Dordrecht: Springer), CDROM, [arXiv:astro-ph/0609647]

Goicoechea, L. J., Shalyapin, V. N., Koptelova, E., et al. 2008, New A, 13, 182 (Paper I)

Hirv, A., Eenmäe, T., Liimets, T., Liivamägi, L. J., \& Pelt, J. 2007, A\&A, 464, 471

Hutchings, J. B. 2003, AJ, 126, 24

Keeton, C. R., Kochanek, C. S., \& Falco, E. E. 1998, ApJ, 509, 561

Kerins, E., et al. 2006, MNRAS, 365, 1099

Kochanek, C.S. 2004, ApJ, 605, 58

Kochanek, C. S., Schneider, P., \& Wambsganss, J. 2004, StrongGravitational Lensing, in Proc. 33rd Saas-Fee Advanced Course, ed. G. Meylan, P. Jetzer, \& P. North (Berlin: Springer)

Kochanek, C. S., et al. 2008, The CfA-Arizona Space Telescope LEns Survey (CASTLES), http: //www.cfa.harvard.edu/glensdata/

Koptelova, E., Shimanovskaya, E., Artamonov, B., et al. 2005, MNRAS, 356, 323

Koptelova, E. A., Oknyanskij, V. L., \& Shimanovskaya, E. V. 2006, A\&A, 452, 37

Kundić, T., Colley, W. N., Gott, J. R., III, et al. 1995, ApJ, 455, L5

Kundić, T., Turner, E. L., Colley, W. N., et al. 1997, ApJ, 482, 75

Magnier, E. A., \& Cuillandre, J. C. 2004, PASP, 116, 449
Manfroid, J., Selman, F., \& Jones, H. 2001, The Messenger, 104, 16 McLeod, B. A., Bernstein, G. M., Rieke, M. J., \& Weedman, D. W. 1998, AJ, 115,1377

Oscoz, A., Alcalde, D., Serra-Ricart, M., et al. 2001, ApJ, 552, 81

Ovaldsen, J. E., Teuber, J., Schild, R. E., \& Stabell, R. 2003a, A\&A, 402, 891

Ovaldsen, J. E., Teuber, J., Stabell, R., \& Evans, A. K. D. 2003b, MNRAS, 345, 795

Paraficz, D., Hjorth, J., Burud, I., Jakobsson, P., \& Elíasdóttir, Á. 2006, A\&A, 455, L1

Pelt, J., Hoff, W., Kayser, R., Refsdal, S., \& Schramm, T. 1994, A\&A, 286, 775 Pelt, J., Kayser, R., Refsdal, S., \& Schramm, T. 1996, A\&A, 305, 97

Pijpers, F.P. 1997, MNRAS, 289, 933

Refsdal, S. 1964, MNRAS, 128, 307

Schild, R. 2005, AJ, 129, 1225

Schmidt, R., \& Wambsganss, J. 1998, A\&A, 335, 379

Schneider, D. P., Hall, P. B., Richards, G. T., et al. 2007, AJ, 134, 102

Sergeev, S. G., Doroshenko, V. T., Golubinskiy, Y. V., Merkulova, N. I., \& Sergeeva, E. A. 2005, ApJ, 622, 129

Serra-Ricart, M., Oscoz, A., Sanchís, T., et al. 1999, ApJ, 526, 40

Smith, J. A., Tucker, D. L., Kent, S., et al. 2002, AJ, 123, 2121

Steele, I. A., Smith, R. J., Rees, P. C., et al. 2004, Ground-based telescopes, ed. J. M. Oschmann, Proc. SPIE, 5489, 679

Stoughton, C., Lupton, R. H., Bernardi, M., et al. 2002, AJ, 123, 485

Ullán, A., Goicoechea, L. J., Muñoz, J. A., et al. 2003, MNRAS, 346, 415

Ullán, A., Goicoechea, L. J., Zheleznyak, A. P., et al. 2006, A\&A, 452, 25

Vanden Berk, D. E., Wilhite, B. C., Kron, R. G., et al. 2004, ApJ, 601, 692

Walsh, D., Carswell, R. F., \& Weymann, R. J. 1979, Nature, 279, 381

Yonehara, A. 1999, ApJ, 519, L31

Yonehara, A., Mineshige, S., Takei, Y., Chartas, G., \& Turner, E. L. 2003, ApJ, 594, 107 